Astronomy

\title{
Two for the price of one
}

This latest image from Subaru, one of the world's largest telescopes, was taken by the Faint Object Camera and Spectograph (FOCAS) during its first night of operation in February. The FOCAS instrument is capable of taking two sorts of pictures: images and spectra, which can be chosen at the flick of a switch. Spectra of faint galaxies provide physical information, such as temperature and density, to complement the optical images.

The image here is of the irregular galaxy M82, which is roughly 12 million light years from Earth. The bluish light running across the image comes from stars in the

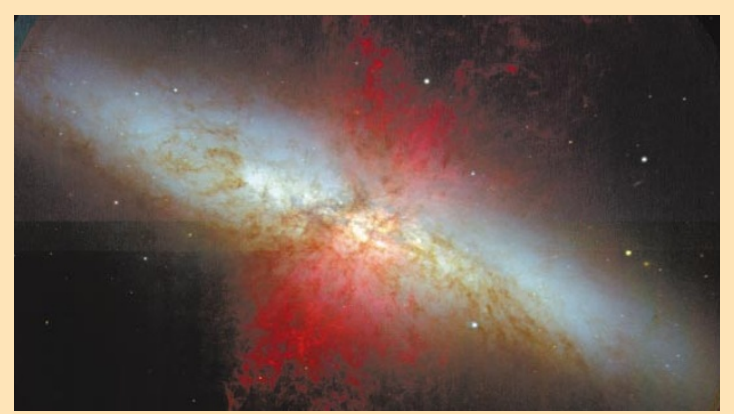

galactic disk. The red filaments flowing out at right angles are produced by ionized hydrogen gas, which is evidence for star formation at the centre of M82.

Subaru - the Japanese name for the constellation Pleiades — was built by Japan's National Astronomical Observatory at the top of
Mauna Kea in Hawaii. The 8.2metre telescope saw its first light early in 1999, but its systems are still being tested and fine tuned. Five of the seven observational instruments have now been added and will soon be ready for routine observations.

Sarah Tomlin cell-cycle progression in vitro. The results of Maiorano et al., showing that the Xenopus Cdt1 protein is required to license DNA by loading the MCM proteins onto it, are consistent with those obtained in S. pombe.

These papers also show that, in both fission yeast and frog, Cdt1 itself associates with DNA. This association is dependent on the presence of the origin-recognition complex $(\mathrm{ORC})^{2}$, as is the association of Cdc6/Cdc18 with $\mathrm{DNA}^{6}$. In fission yeast extracts, Cdt1 and Cdc6/Cdc18 can be precipitated together by an antibody against just one of the proteins, suggesting that they might form a complex. However, they can also associate with DNA independently of one another. These results indicate that, early in the G1 phase of the cell cycle (the stage preceding $S$ phase), Cdt1 and Cdc6/Cdc18 may bind together to ORCs on DNA (Fig. 1a), where they are required for licensing to occur (Fig. 1b).

A further essential licensing activity, termed RLF-B, is probably also required at this stage ${ }^{9}$. The protein underlying RLF-B activity is unknown, and could theoretically correspond to Cdt1. However, the behaviour of RLF-B differs from that of Cdt1: unlike Cdt1, RLF-B activity does not associate with unlicensed DNA 9 . Cdt1 also seems to behave differently from $\mathrm{Cdc} 6 / \mathrm{Cdc} 18$, as, in the frog system, Cdt1 remains associated with DNA right up until the start of $S$ phase (Fig. 1d), whereas Cdc6/Cdc18 is displaced from DNA immediately after origins are licensed ${ }^{10}$ (Fig. 1c). Further studies will be needed before we fully understand the roles of these proteins in origin licensing.
The existence of this complex series of events in origin licensing probably reflects the essential function of this system in maintaining genomic integrity. And it is probably even more important to prevent the relicensing of replicated origins (and hence the re-initiation of DNA replication) in multicellular organisms than it is in single-celled ones. The occasional re-replication event would have little effect on the proliferative capacity of a strain of single-celled organisms such as yeast. But, by generating a cancer-causing mutation, it might be fatal to a multicelled organism. Re-assembling the Cdt1 protein - which is regulated in different ways (transcriptionally or post-translationally) in yeast ${ }^{1}$ and frogs ${ }^{2}$ - at replication origins represents yet another step that is necessary for re-licensing to occur. We can expect the regulation of the replication licensing system to become more complex still.

J. Julian Blow and Shusuke Tada are at the CRC

Chromosome Replication Research Group,

Department of Biochemistry, University of Dundee, Dow Street, Dundee DD1 5EH, UK.

e-mail: j.j.blow@dundee.ac.uk

1. Nishitani, H., Lygerou, Z., Nishimoto, T. \& Nurse, P. Nature 404, 625-628 (2000).

2. Maiorano, D., Moreau, J. \& Méchali, M. Nature 404, 622-625 (2000).

Blow, J. J. \& Laskey, R. A. Nature 332, 546-548 (1988).

4. Tye, B. K. Annu. Rev. Biochem. 68, 649-686 (1999).

5. Hofmann, J. F. \& Beach, D. EMBO J. 13, 425-434 (1994).

6. Coleman, T. R., Carpenter, P. B. \& Dunphy, W. G. Cell 87, 53-63 (1996).

Kelly, T. J. et al. Cell 74, 371-382 (1993).

8. Nishitani, H. \& Nurse, P. Cell 83, 397-405 (1995).

9. Tada, S., Chong, J. P. J., Mahbubani, H. M. \& Blow, J. J. Curr. Biol. 9, 211-214 (1999)

10. Rowles, A., Tada, S. \& Blow, J. J. J. Cell Sci. 112, 2011-2018 (1999).

\section{Daedalus}

\section{The sharper image}

Last week Daedalus proposed to use silicon microfabrication to make klystrons with a resonant cavity only a wavelength of light or so across. Such resonant sources could generate visible light directly. For useful brightness, many such cavities would have to be formed on a single silicon chip: perhaps ten thousand per square centimetre. Such a chip could be a wonderful source of intense, coherent, steerable light.

If all the photoklystrons were identical, and spaced as an even array, their outputs would combine in phase to give a coherent, parallel beam. Even the most exact microfabrication could not do this by itself. But a klystron can be fine-tuned by adjusting its electrode voltages, a simple task for the control circuitry integrated on the same chip. With all the klystrons thus phased together, they would form a collective source as parallel and monochromatic as any laser, but far more efficient, and with a useful tunable range.

Even better, by shifting the phase stepwise across the array, the light could be steered at electronic speed, like a radar beam. DREADCO's 'Photophasor' lamp could give 'lidar' the fast scanning power of the best radar. But its most important use will surely be in large-scale imaging. Driven by standard TV scanning circuitry, the Photophasor will be able to draw a bright moving image on a big screen. With red, green and blue klystrons spaced on the chip like the tricolour matrix of dots on a television screen, it could write a big bright full-colour motion picture under direct digital control.

Electronic cinema and big-screen display will be transformed. The clumsy, blurry cathode-ray-tube projector will be swept away by the simple, elegant Photophasor. Highly efficient, it will be a cool, low-power device, interfacing directly with TV or computer inputs. Cinemas will abandon film for DVD disks screened by Photophasor, and Photophasor business presentations will have even more compulsive visual gorgeousness to disguise their lack of ideas. And by scaling down the technology, a simple Photophasor display could even displace the ubiquitous cathode ray TV and computer screen. The crippling limit of the personal computer, the low resolution and limited area of its display, would be overcome at last.

David Jones

The Further Inventions of Daedalus (Oxford University Press), 148 past Daedalus columns expanded and illustrated, is now on sale. Special Nature offer: m.curtis@nature.com 